Brief Report

\title{
MiR-325-3p functions as a suppressor miRNA and inhibits the proliferation and metastasis of glioma through targeting FOXM1
}

\author{
Qijiang Xiong ${ }^{1}$, Hai Su${ }^{1, *}$ \\ ${ }^{1}$ Department of Neurosurgery, Yongchuan Hospital ofChongqing Medical University, Yongchuan, 402160 Chongging, China \\ *Correspondence: 700222@hospital.cqmu.edu.cn (Hai Su) \\ DOI:10.31083/j.jin2004103 \\ This is an open access article under the CC BY 4.0 license (https://creativecommons.org/licenses/by/4.0/). \\ Submitted: 8 October 2021 Revised: 8 November 2021 Accepted: 11 November 2021 Published: 30 December 2021
}

\begin{abstract}
Clioma is a malignant brain tumor exhibiting high levels of proliferation and metastasis, and these have been related to its poor prognosis and high mortality rate. MicroRNA (miRNA)-325-3p exhibits tissue-specific expression profiles and is aberrantly expressed in multiple types of malignant tumors. Our research focuses on determining the function and mechanism of action of miR-325-3p in glioma. The relative expression levels of miR-325-3p in glioma tumor tissues and cell lines were verified by qRT-PCR. The effect of 325 -3p on glioma tumor cell behavior was assessed using CCK-8 assays, EDU staining, colony formation assays, flow cytometry, transwell invasion assays, and a xenograft model. In addition, we searched for miR-325-3p targets, and their potential mechanism of action was demonstrated using a reporter assay and rescue experiments. Results showed that the expression levels of miR-325-3p in glioma cancer tissues and tumor cell lines were significantly lower than that of normal paired adjacent tissue or normal cell lines. Functional experiments illustrated that tumor proliferation, migration and invasion were suppressed via upregulation of miR-325-3p. To assess whether FOXM1 is a target of miR-325-3p, we examined this hypothesis using a luciferase report assay and then found that miR-325-3p could modulate the expression of FOXM1. Furthermore, the functional role of miR-325-3p was also confirmed in a xenograft model using nude mice. Together, our data demonstrated that in glioma, miR-325-3p may inhibit cancer cell growth through the suppression of FOXM1 and could be a promising new target for treating this type of brain cancer.
\end{abstract}

Keywords

MiR-325-3p; FOXM1; Proliferation; Metastasis; Clioma; Neuro-oncology

\section{Introduction}

Glioma is one of the most malignant tumors and accounts for more than $80 \%$ of all primary malignant tumors of the central nervous system [1]. The median overall survival rate is constant at 16 months, although huge progress has been made in therapy [2]. At present, surgery, chemotherapy, and radiotherapy are the main strategies for glioma treatment [3]. However, this disease is virtually incurable, and its five-year survival rate remains unsatisfactorily low [4]. Thus, it is key to understand the molecular mechanisms for glioma's occurrence and progression, which may help uncover new therapeutic targets. Recent studies have revealed many abnormally expressed genes, which may be involved in glioma progression [5]. MiRNAs are endogenous, non-coding RNAs that regulate multiple physiological and pathological processes at the posttranscriptional level [6-8] and mounting evidence suggests the involvement of miRNAs in multiple cancers, including glioma. Here, we specifically focused on miR-325-3p, which was found to be aberrantly expressed in multiple types of malignant tumors. For example, miR-325-3p showed low expression levels in colorectal cancer (CRC), representing a key regulator of bone metastasis [9]. In gastric cancer (GC), the miR-325-3p was decreased, and its low expression level was also associated with metastasis [10]. Furthermore, miR325-3p has an effective therapeutic function in treating lung and bladder cancer $[11,12]$. However, the exact functions of miR-325-3p in glioma have yet to be determined, and thus these data suggest that miR-325-3p could be a potential therapeutic target for malignant glioma (Fig. 1).

\section{Materials and methods}

\subsection{Human tissues}

Twenty-four glioma tissue samples and adjacent normal tissue samples were obtained from Yongchuan Hospital of Chongqing Medical University, and all written informed consent was obtained from the patients. At least two pathologists diagnosed and confirmed all tissues had not been treated with radiotherapy or chemotherapy before surgery. The tissues were collected and kept at $-80{ }^{\circ} \mathrm{C}$ until analysis, and the Ethics Committee of Yongchuan Hospital of Chongqing Medical University approved this research.

\subsection{Cell culture and transfection}

Normal human astrocytes (NHAs) and glioma cell lines SW1783, U87, and LN229 were purchased from the Chinese Academy of Sciences cell bank. All Cells were cultured using DMEM and RPPI-1640 (Gibco, Carlsbad, CA, USA), 10\% fetal bovine serum (Gibco) in an incubator at $37{ }^{\circ} \mathrm{C}, 5 \% \mathrm{CO}_{2}$, and $100 \%$ humidity. MiR-325-3p mimics (miR-325-3p-mimic), miR-325-3p inhibitors (miR-3253p-inhibitor), miR-325-3p negative controls (miR-325-3p$\mathrm{NC}$ ), and lentivirus containing miR-325-3p or its control 


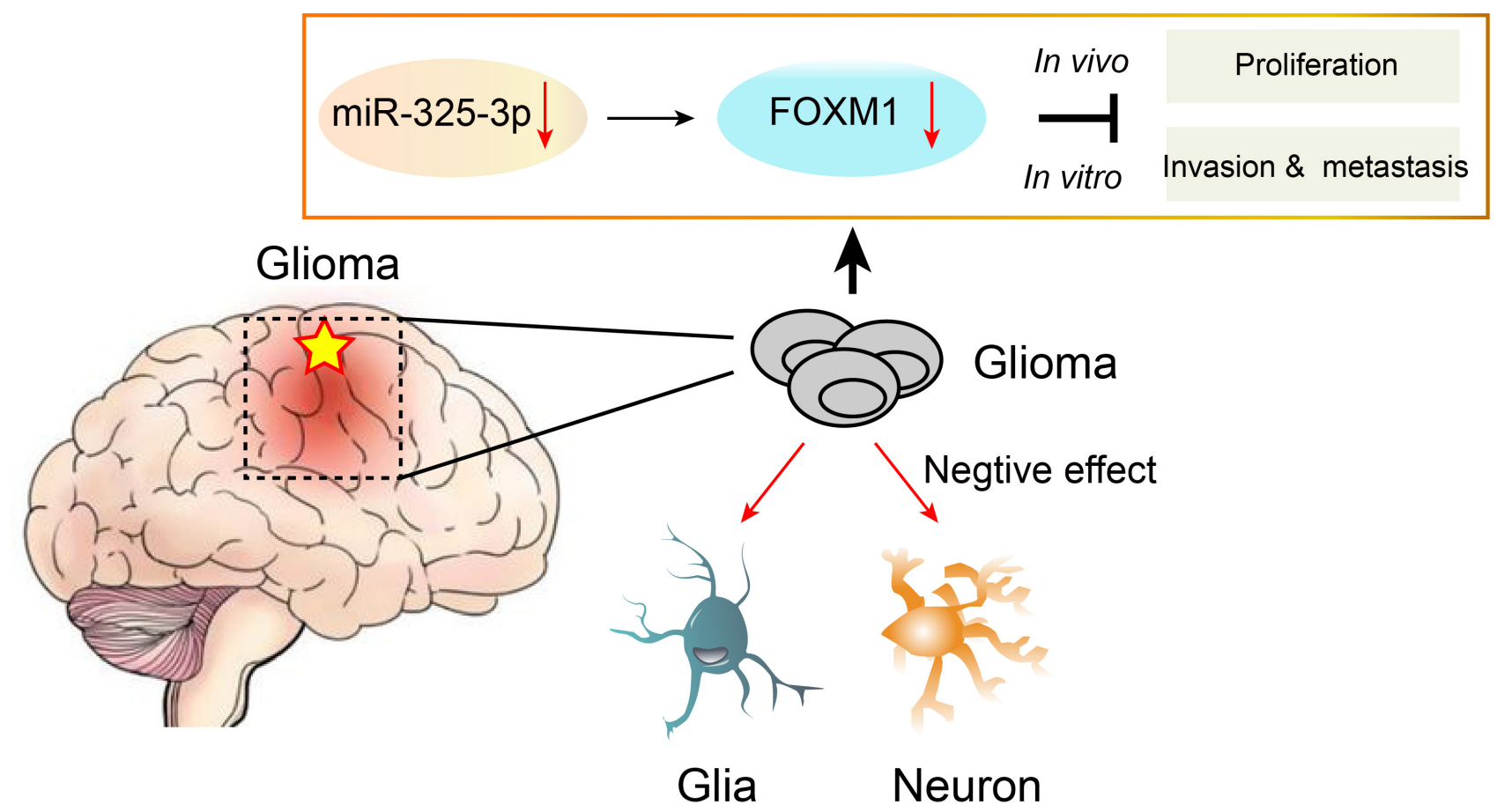

Fig. 1. Schematic illustration of rationale to show the intersections between neuroscience and glioma biology.

were designed and synthesized by Wuhan Genesil Biotechnology Co., Ltd (Wuhan, China) and transfected using Lipofectamine transfection reagent (Invitrogen, MA, USA) according to the manufacturer's instructions.

\section{$2.3 q R T-P C R$}

Cells or tissues were collected, and total RNA was extracted with TRIZOL reagent. Then total miRNAs were isolated using the mirVana ${ }^{\mathrm{TM}}$ miRNA Isolation Kit. Samples were reverse transcribed into cDNA using a Revert Aid First Strand cDNA Synthesis Kit (RR047AA, TaKaRa, Japan) and TaqMan ${ }^{\text {TM }}$ MicroRNA Reverse Transcription Kit (miRNA) (4366596, Invitrogen, USA). After the reverse transcription, products were diluted two-fold, and qRT-PCR was performed using: $2 \mu \mathrm{L}$ of cDNA added to $10 \mu \mathrm{L}$ of SYBR Premix Ex TaqIITM $(2 \times)($ RR420A, TaKaRa, Japan) and 0.2 $\mathrm{mol} / \mathrm{L}$ of each primer. Finally, sterile distilled water was used to complete the reaction to $20 \mu \mathrm{L}$. The reaction was cycled and analyzed on an ABI 7500 Fast Real-Time PCR system (Biosystems, USA). The reaction conditions were as follows: $95^{\circ} \mathrm{C}, 3 \mathrm{~min}$; 40 cycles: $95^{\circ} \mathrm{C}, 30 \mathrm{~s} ; 60^{\circ} \mathrm{C}, 15 \mathrm{~s}$ and the data were analyzed using the $2^{-\Delta \Delta C t}$ method. The mRNA expressions of FOXM1 were normalized to GAPDH, while miR-325-3p was normalized to U6. The following primers were used:

$\begin{array}{lr}\text { miR-325-3p, forward: } & 5^{\prime}- \\ \text { GCGCCTAGTAGGTGTCCAGT-3' and } & \\ \text { reverse: } \text { 5'-CTCAACTGGTGTCGTGGAGTC-3' }^{\prime} & \\ \text { FOXM1, forward: } & 5^{\prime}- \\ \text { GGTGCCTACTGTGGATATAGCCG-3' and } & \\ \text { reverse: } \text { 5'-GTTGCTGAGTAGGCAGTAGCCGA-3' }^{\prime}\end{array}$

U6, forward: 5'-CTCGCTTCGGCAGCACA-3' and reverse: $5^{\prime}$-AACGCTTCACGAATTTGCGT-3'

GAPDH, forward: $5^{\prime}$ GGAGCGAGATCCCTCCAAAAT $-3^{\prime}$ and reverse: 5'-GGCTGTTGTCATACTTCTCATGG-3' .

\subsection{Cell viability assay}

For cell viability assays [13], a CCK-8 kit (C0038, Beyotime Biotechnology, China) was used according to the manufacturer's instructions. Transfected cells were inoculated into 96-well plates and incubated overnight. Add CCK-8 reagent $10 \mathrm{~mL}$ to each well, and incubate at $37^{\circ} \mathrm{C}$ for 2 hours. The absorption value was measured at $450 \mathrm{~nm}$ with a microplate analyzer.

\subsection{EDU incorporation assay}

Twenty $\mu \mathrm{M}$ of EDU was used to label cells for 15 minutes at $37^{\circ} \mathrm{C}$, and they were then fixed with $4 \%$ paraformaldehyde for 10 minutes, followed by denaturation with $\mathrm{HCl}(\mathrm{pH}=2)$ at $37^{\circ} \mathrm{C}$ and neutralized in $20 \mathrm{mM} \mathrm{Na}_{2} \mathrm{CO}_{3}$ for 1 hour. Next, the cells were blocked with $5 \%$ bovine serum albumin (BSA) for 30 minutes and immunostained with anti-EDU (1:500) primary antibody overnight. Secondary antibodies were then used at 1:1000 dilution, and DAPI (1:1000, Sigma) was added during secondary antibody incubation.

\subsection{Colony formation assay}

Cells were plated in 6-well plates (500 per/well), and the medium was replaced every 3-4 days. When cell colonies were formed after 2 weeks, they were fixed and stained. Visible colonies were photographed and counted. 


\subsection{Transwell assays}

As previously described, cell invasion assays were performed using the Bio-Coat Cell Migration Chamber (BD Biosciences, MA, USA) [14]. Then a 24-well plate containing an $8 \mu \mathrm{m}$-pore filter cell culture insert was used. Before the invasion experiment, $70 \mu \mathrm{L}$ of matrix glue (diluted with DMEM: 1:4) was spread on the bottom of the upper chamber and placed in an incubator for 3 hours. The invasion assay was as follows: transfected cells $\left(3 \times 10^{5}\right.$ cells/ $\left.500 \mu \mathrm{L}\right)$ were resuspended in DMEM and transferred to the upper chamber of the transwell plate. DMEM containing 20\% FBS was placed in the transwell plate chamber $(500 \mu \mathrm{L})$, and after incubation $\left(5 \% \mathrm{CO}_{2}, 37^{\circ} \mathrm{C}\right)$ for 24 hours, the non-transfected cells were rubbed with cotton swab. The invasive cells were then fixed with 4\% paraformaldehyde (P0099, Beyotime Biotechnology, China) and stained with crystal violet (C0121, Beyotime Biotechnology, China). Cell counts were randomly selected from five microscope fields $(100 \times)$ (NIB620, Boshida, China).

\subsection{Immunohistochemistry}

After the sections were rehydrated as above [15], heatinduced antigen retrieval was performed in sodium citrate for 15 minutes in a $95{ }^{\circ} \mathrm{C}$ water bath, followed by the addition of $3 \%$ hydrogen peroxide and 5\% BSA to block endogenous peroxidase activity and non-specific antigens. Next, the sections were incubated with primary antibodies targeted against FOXM1, Ki67, and $N$-cadherin (Abcam) at 4 ${ }^{\circ} \mathrm{C}$ overnight. The sections were incubated in HRP conjugated secondary antibodies the next day, and sections were visualized using DAB and imaged using a bright field microscope.

\subsection{Dual-luciferase activity assay}

The interactions between miR-325-3p and FOXM1 were assessed using TargetScan tools. The 3'-UTR. FOXM1 fragment was cloned full-length into a pmirGLO expression vector (Promega, WI, USA). The luciferase vector and miR-325$3 \mathrm{p}$ mimics were cotransfected into U87 cells the and the luciferase activity was measured with a Dual-Luciferase Assay System (Promega, Madison, WI, USA) 48 h later.

\subsection{Western blotting}

Total protein was extracted with RIPA reagent (P0013B, Beyotime Biotechnology, China), and the protein concentration was measured using a BCA protein detection kit (P0012, Beyotime Biotechnology, China). Equal amounts of total protein $30 \mu \mathrm{g}$ were then loaded with size markers (PR1910, Solarbio, China), and 10\% SDS-PAGE was performed. Then, proteins were transferred to a PVDF membrane and blocked with $5 \%$ skimmed milk for 1 hour at room temperature and washed with TBST for 5 minutes. The blocked membrane was incubated overnight at $4{ }^{\circ} \mathrm{C}$ with primary antibody (FOXM1, 1:2000; Abcam, MA, USA; E-cadherin, 1:3000; Abcam; $N$-cadherin, 1:4000; Abcam; vimentin, 1:4000; Abcam; Fibronectin, 1:5000; Abcam; GAPDH, 1:4000, Abcam) and washed four times ( $5 \mathrm{~min}$ each), followed by in- cubation with secondary antibody (1:5000, Abcam) at room temperature for 1 hour. After further TBST washes, protein bands were detected using an enhanced chemiluminescence kit (WBKLS0500, MILLIPORE, USA) and enhanced Chemiluminescence Advanced System (Bio-Rad, CA, USA) and quantified with Image $J$ software (National Institutes of Health, MD, USA). In addition, GAPDH served as an internal control for quantifying the target proteins.

\subsection{Glioma xenograft mouse models}

The Animal Care Committee approved the animal experiments of Yongchuan Hospital of Chongqing Medical University. All experiments involving mice were conducted in accordance with the guidelines for animal welfare formulated by the laboratory animal center at Yongchuan Hospital of Chongqing Medical University. The nude mice aged 6 weeks were purchased from Chongqing Medical University Experimental Animal Center. Each mouse was injected subcutaneously with $6 \times 10^{5}$ U87 cells transfected with miR325-3p suspended in $200 \mu \mathrm{L}$ of Hanks' balanced salt solution. The tumor size in each animal was measured every 7 days. On day 21, the mice were sacrificed, and xenografts were excised, weighed, photographed, paraffin-embedded and sectioned for $\mathrm{HE}$ and immunohistochemical staining.

\subsection{Statistical analysis}

Data were assessed using Student's $t$-test or one-way ANOVA in GraphPad Prism 7.0 (GraphPad, CA, USA) software and expressed as mean \pm standard error of the mean (SEM). $p$-value $<0.05$ was considered statistically significant.

\section{Results \\ 3.1 Decreased miR-325-3p expression in glioma tissues and cell lines}

To determine the expression profile of $\mathrm{miR}-325-3 \mathrm{p}$ in glioma tissues, more than twenty-paired surgical glioblastoma and RT-qPCR analyzed para-carcinoma tissues. It can be seen that miR-325-3p expression was significantly decreased in glioma tissues compared to the adjacent normal tissues (Fig. 2A, $p<0.01$ ). We further performed RT-qPCR analysis of $\mathrm{miR}-325-3 \mathrm{p}$ expression in three human glioma cell lines SW1783, U87, LN229, and an astrocyte cell line NHA. As expected, miR-325-3p levels were lower in the glioma cell lines when compared to the astrocytes (Fig. 2B). These results suggested that miR-325-3p is a promising prognostic biomarker for glioma and may contribute to malignant progression.

\subsection{In vitro upregulation of miR-325-3p inhibited glioma cell viability and proliferation}

As an association between miR-325-3p and glioma has been confirmed, we next investigated the functional roles of miR-325-3p in glioma cell growth. MiRNA-mimics were specifically and effectively used to upregulate miR-325-3p expression in SW 1783 and U87 glioma cell lines (Fig. 3A). Furthermore, the CCK8 assay showed that upregulation of miR- 
A

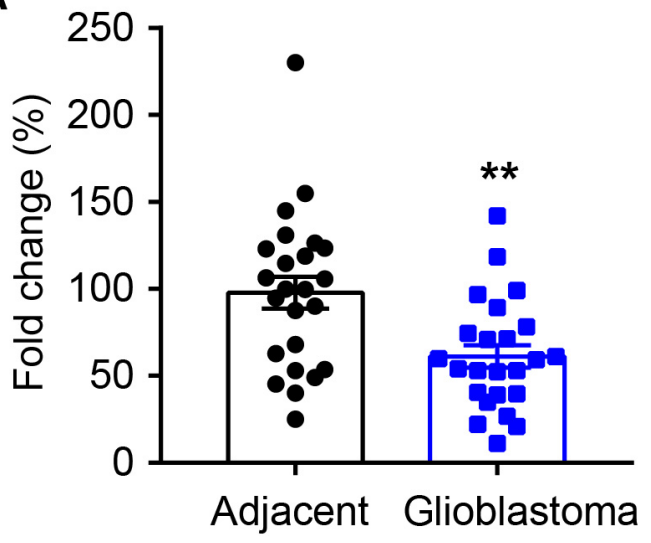

B

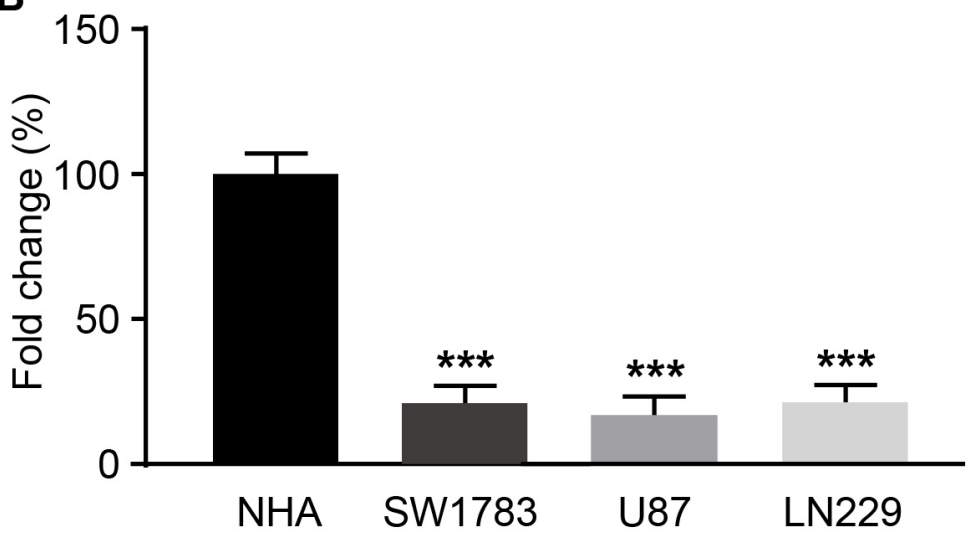

Fig. 2. Decreased miR-325-3p expression in glioma tissue and cell lines. (A) Results from RT-qPCR analysis showed that miR-325-3p was decreased in human glioma tissues. (B) Decreased miR-325-3p levels were also detected in glioma cells. Data are presented as the mean \pm SEM, ${ }^{* *} p<0.01,{ }^{* * *} p<0.001$, compared to the corresponding control group.

A

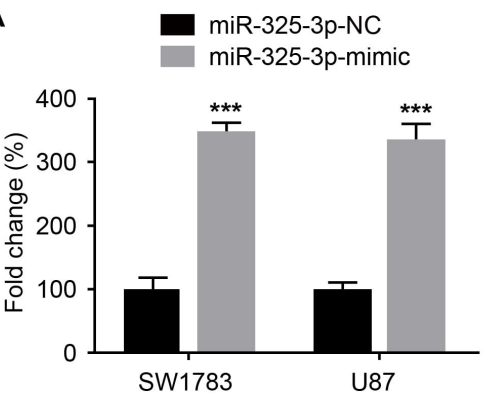

C



B

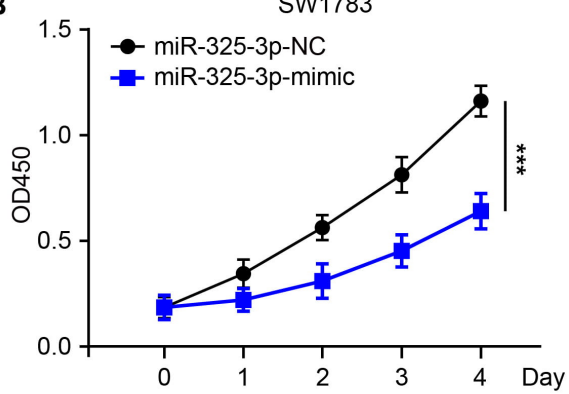

D

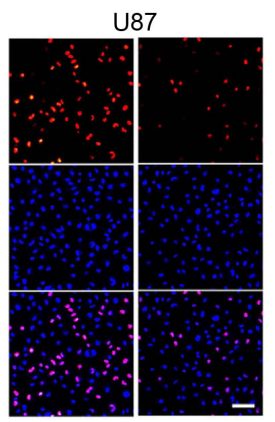

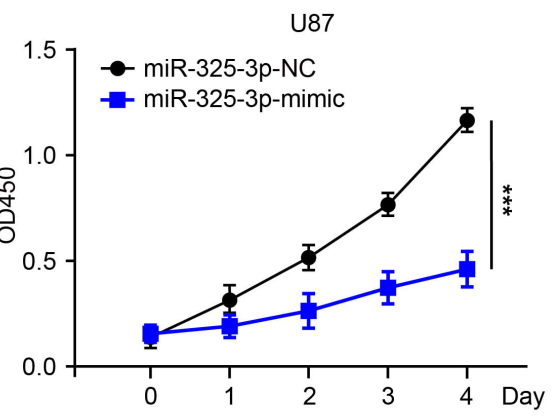

miR-325-3p-NC miR-325-3p-mimic



Fig. 3. In vitro upregulation of miR-325-3p inhibits cell growth. (A) The transfection efficiency of miR-325-3p was evaluated using RT-qPCR. (B) Cell viability of SW 1783 and U87 cells transfected with the miR-325-3p-mimic was evaluated by CCK8 assay. (C) EDU assay was conducted to examine proliferation. (D) Colony formation assay for the detection of proliferation. Data are presented as the mean \pm SEM, ${ }^{* * *} p<0.001$, compared to the miR-325$3 \mathrm{p}-\mathrm{NC}$ group.

325-3p inhibited the proliferation rate of SW1783 and U87 cells (Fig. 3B). Using the EdU and colony formation assays, we also found that upregulation of miR-325-3p inhibited the growth of SW 1783 and U87 cells (Fig. 3C,D). These data showed that miR-325-3p upregulation could effectively inhibit glioma cell proliferation.

\subsection{Upregulation of miR-325-3p decreased glioma cell migration and invasion}

Metastasis is a major risk for patients with glioma, and therefore, to further determine the function of miR-325-3p in glioma cell migration and invasion in vitro, transwell assays were employed. We found that upregulation of miR-3253 p significantly inhibited the migration of SW 1783 and U87 cells (Fig. 4A,B). The transwell assay also consistently indicated that overexpression of miR-325-3p also decreased their 
A

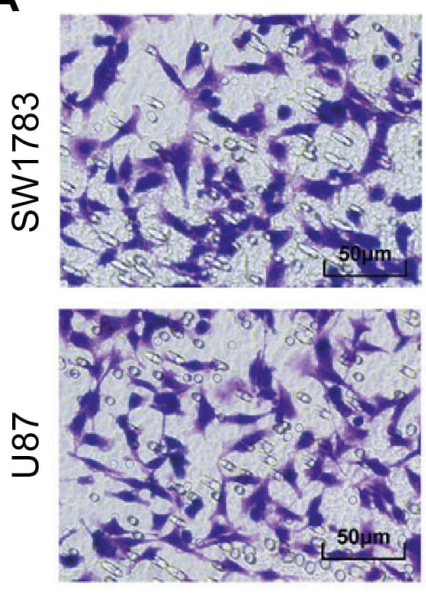

miR-325-3p-NC

C
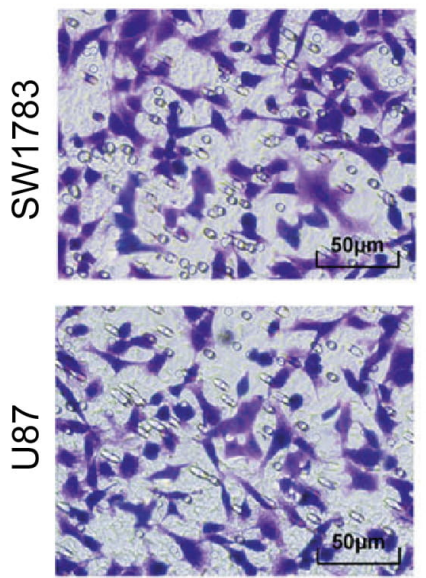

miR-325-3p-NC
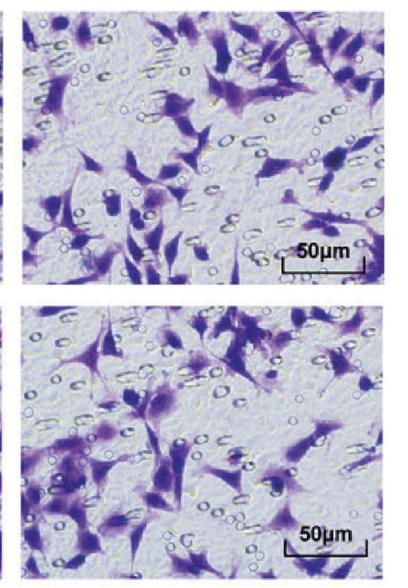

miR-325-3p-mimic

\section{B}
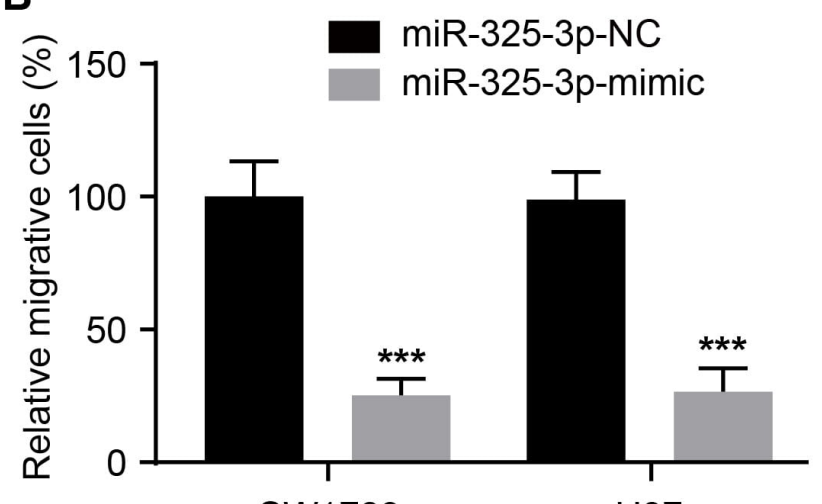

SW1783

U87
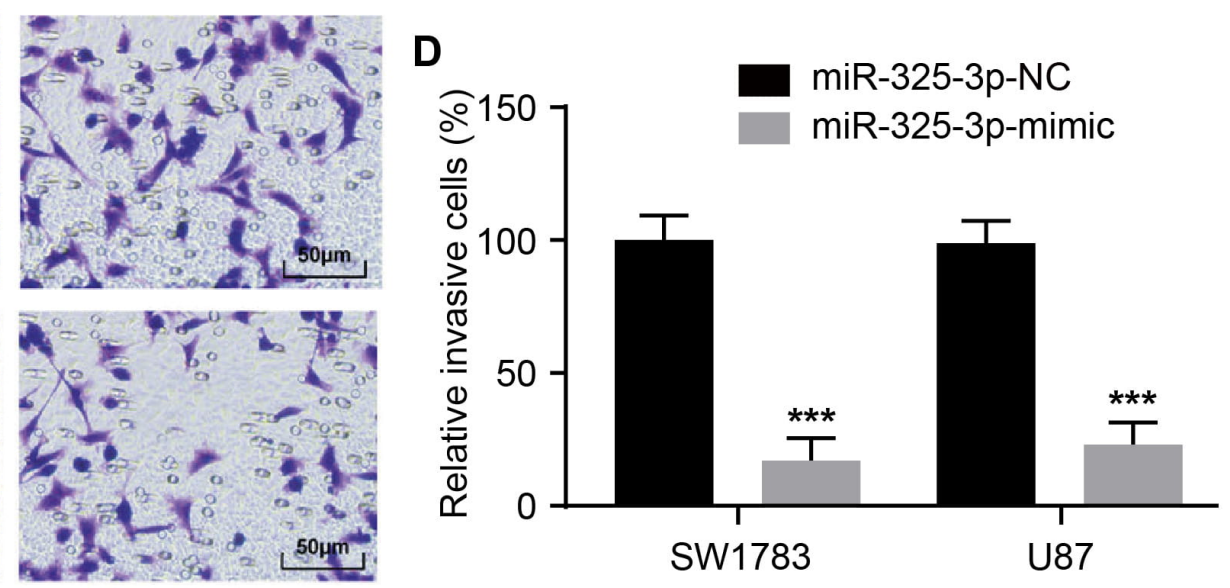

Fig. 4. Upregulation of miR-325-3p suppressed glioma cell migration and invasion. (A,B) Transwell assay revealed that upregulation of miR-325-3p decreased the migration capability of SW 1783 and U87 cells. (C,D) Transwell assay revealed that upregulation of miR-325-3p decreased the invasion capability of SW1783 and U87 cells. Data are presented as the mean \pm SEM, ${ }^{* * *} p<0.001$, compared to the miR-325-3p-NC group.

invasive capability (Fig. 4C,D). Such findings suggested that miR-325-3p suppressed the malignant behaviors of glioma cells.

\subsection{FOXM1 is a direct target for miR-325-3p}

Cumulative evidence indicates that FOXM1 serves as a tumor promoter or suppressor in multiple tumors, regulating a wide range of biologic processes [16-18]. Through bioinformatics software TargetScan (http://www.targetscan.org) analysis, we predicted the potential targets of miR-325-3p and found that FOXM1 may represent such a target. FOXM1 does not interact with any known miR-325-3p targets [912]. After the histological analysis, immunohistochemistry revealed that FOXM1 expression was increased in glioma tissue (Fig. 5A), and as shown in Fig. 5B, FOXM1 expression was inversely correlated with miR-325-3p. Thus, both wild-type (WT) and mutant (Mut) luciferase reporters for FOXM1 were generated. Results showed that miR-325-3p suppressed activity in the Foxm1-WT group, but the opposite result was seen for U87 cells (Fig. 5C), thus confirming an interaction. Moreover, western blot analysis revealed that transfection with the miR-325-3p mimic or inhibitor significantly increased/decreased FOXM1 protein levels' expression compared to the control (Fig. 5D,E). These results confirmed a role for miR-325-3p in the regulation of FOXM1 in glioma cell lines.

\subsection{FOXM1 is involved in the miR-325-3p induced migration and invasion of glioma cells}

A series of rescue assays were performed to determine whether FOXM1 expression accounted for miR-325-3pmediated migration and invasion in glioma cells. U87 cells were cotransfected with miR-325-3p-NC or miR-325-3pmimic and pcDNA3.0 plasmid containing control or FOXM1 plasmid, and our western blot results confirmed the effectiveness of this rescue strategy (Fig. 6A). As expected, overex- 
A


C

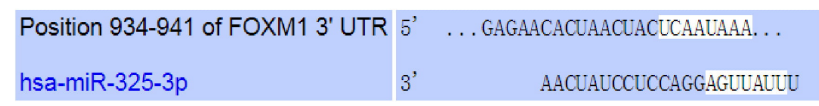

WT FOXM1 3' UTR $5^{\prime}$...GAGAACACUAACUACUCAAUAAA...

IIIIII|

miR-325-3p

3' AaCUAUCCUCCAgGaGUUAUUU

:: : :

Mut FOXM1 3' UTR 5' ...GAGAACACUAACUACAUCUUCCA.

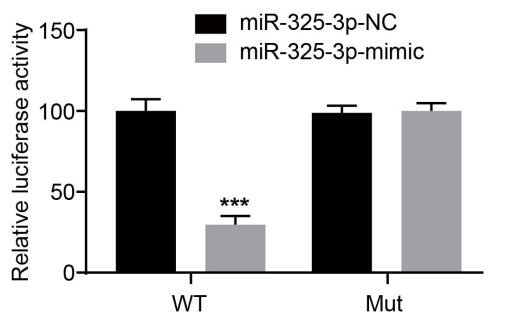

D

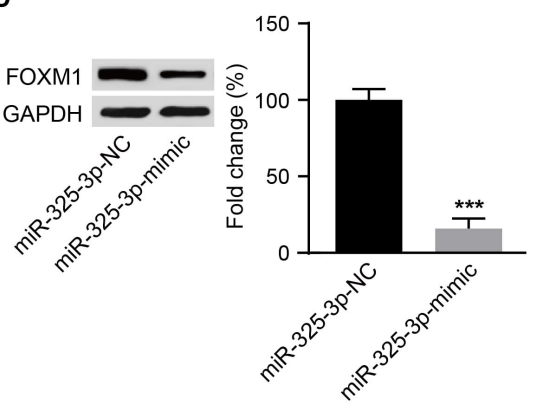

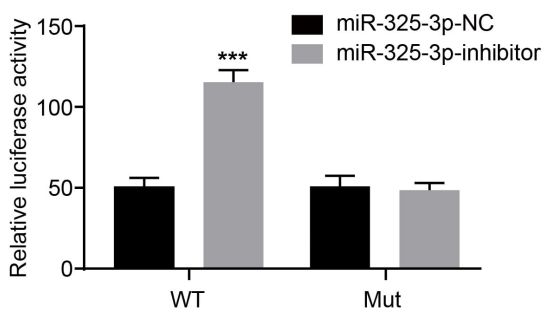

E

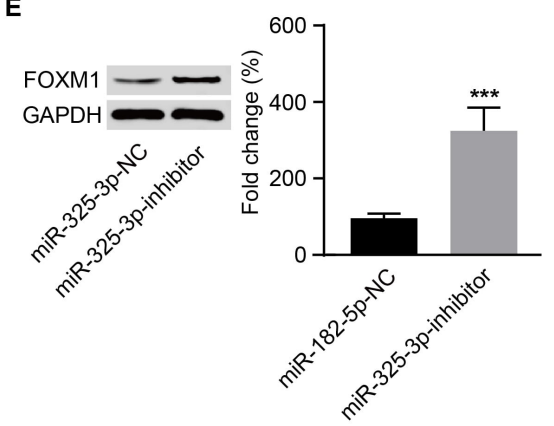

Fig. 5. FOXM1 is a direct target for miR-325-3p in glioma. (A) Tissue comparison using H\&E staining and IHC, showing higher levels of FOXM1 in glioma tissues. (B) An inverse relationship between FOXM1 and miR-325-3p in glioma tissues. (C) FOXM1 was predicted to be a target for miR-325-3p, based on the Targets can database, and the WT and Mut luciferase reporter plasmids were generated to perform dual-luciferase reporter assays. (D,E) The expression of FOXM protein after transfection with NC, mimic, or inhibitor in U87 cells. Data are presented as the mean \pm SEM, ${ }^{* * *} p<0.001$, compared to the miR-325-3p-NC group.

pression of FOXM1 attenuated the inhibitory effect of miR325-3p on migration (Fig. 6B,C) and invasion (Fig. 6D,E). Furthermore, cancer cell invasion and migration are usually associated with epithelial-mesenchymal transition (EMT). For this reason, we further explored proteins associated with the EMT pathway. We found that FOXM1 overexpression reversed the effect of miR-325-3p upregulation, causing a decrease in E-cadherin expressions and an increase in $\mathrm{N}$ cadherin, Vimentin, and Fibronectin in U87 cells (Fig. 6F). Remarkably, FOXM1 overexpression reversed the suppressive effect induced by miR-325-3p upregulation in U87 cells.

\subsection{Upregulation of miR-325-3p repressed glioma cell growth in vivo}

To validate our results in vivo, nude BALB/c mice were injected in the left flanks with NC or miR-325-3poverexpressing U87 cells. Tumor volumes and weights in the miR-325-3p-overexpressing group were significantly reduced compared to the NC group (Fig. 7A,B). Furthermore, $\mathrm{H} \& \mathrm{E}$ staining and immunohistochemistry for the proliferation marker, Ki67, indicated that upregulation of miR-325$3 \mathrm{p}$ inhibited glioma proliferation in vivo (Fig. 6C). IHC staining showed that the EMT-associated marker $N$-cadherin was significantly lower in the miR-325-3p-overexpressing group when compared to the NC group, which is consistent with our in vitro results (Fig. $7 \mathrm{C}$ ). Thus, these results demonstrated that miR-325-3p could inhibit glioma cell growth in vivo.

\section{Discussion}

Glioma is one of the most aggressive and terminal diseases associated with the central nervous system and is associated with a very poor median survival of fifteen months. Therefore, there is an urgent need to uncover more biomarkers to enable clinicians to predict when and what therapy to deploy and help determine prognosis [19]. A growing number of studies have focused on the role of miRNAs in tumorigenesis, as they are important for many biological processes, such as cell proliferation, differentiation and invasion in a variety of cancer types. The loss of miR-325-3p expression has been reported in CRC and gastric cancer $[9,10]$.

These results have highlighted miR-325-3p as a potential new tumor regulatory molecule. However, the mechanism involved in the regulation of glioma growth by miR-325-3p remains unknown. We firstly detected the downregulation of miR-325-3p in glioma tissues and cells. We then confirmed the inhibitory effect of miR-325-3p on proliferation 
A
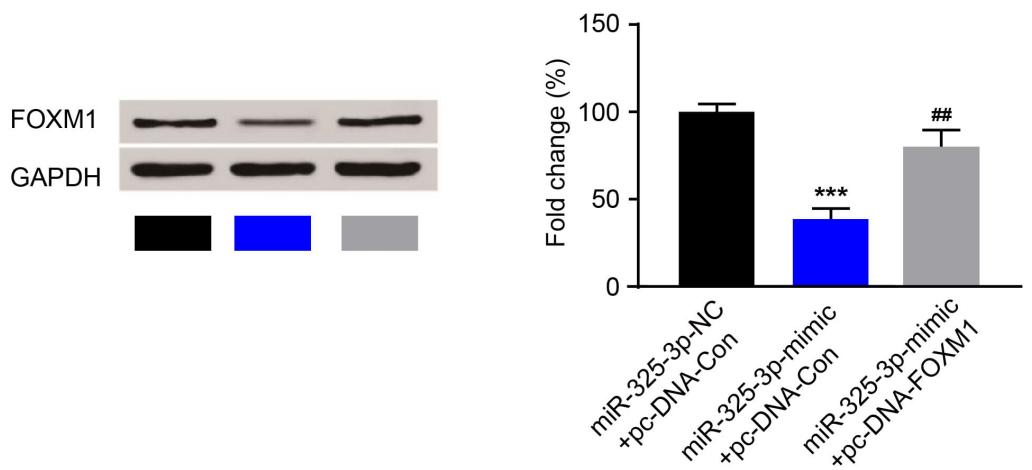

B
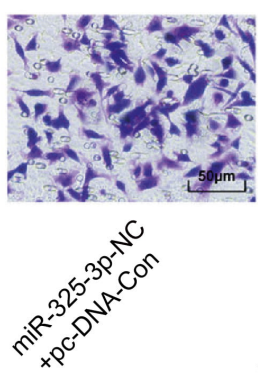

תरe

0
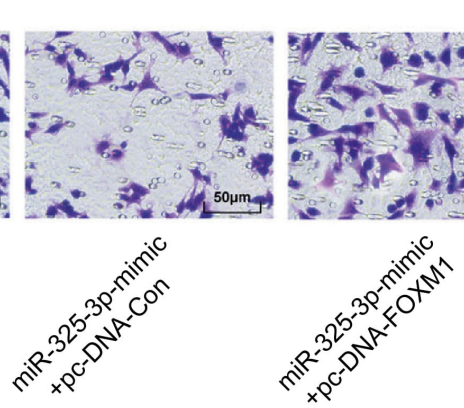

C
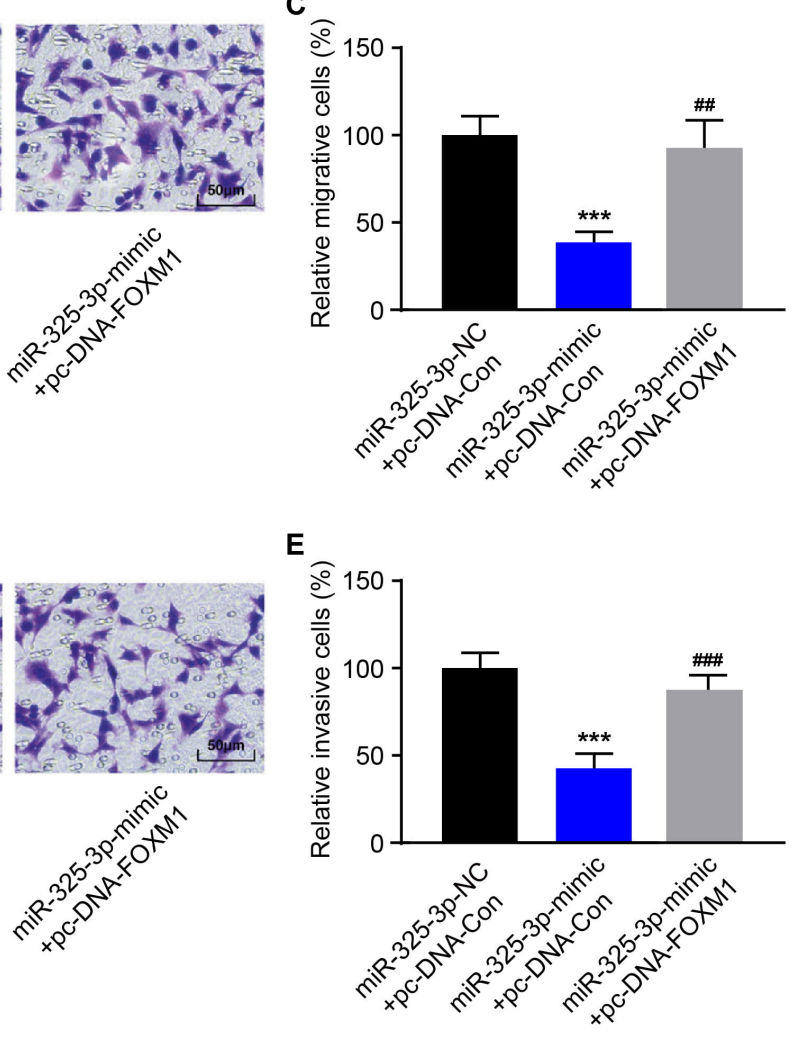
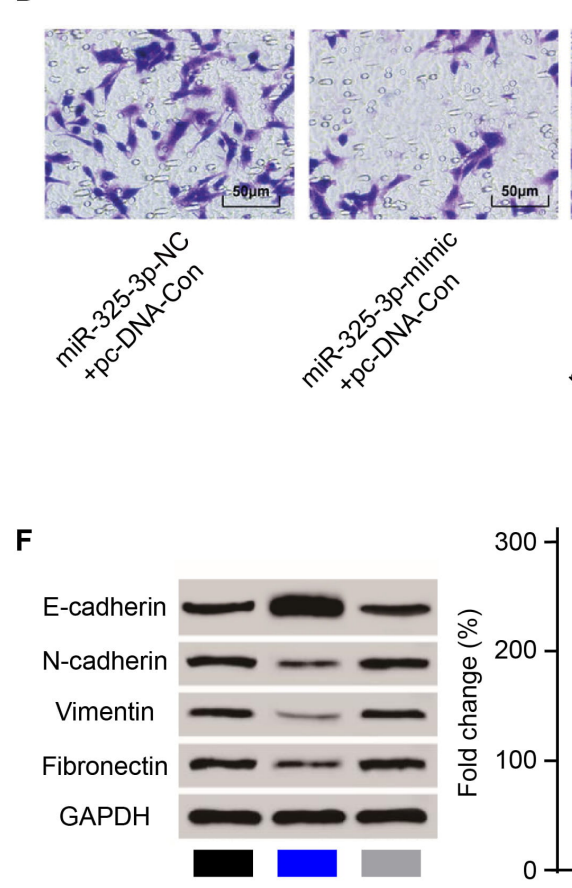

$\square \begin{aligned} & \text { miR-325-3p-NC } \\ & +p c-D N A-C o n\end{aligned}$

miR-325-3p-mimic

+ pc-DNA-Con

miR-325-3p-mimic

+pc-DNA-FOXM1

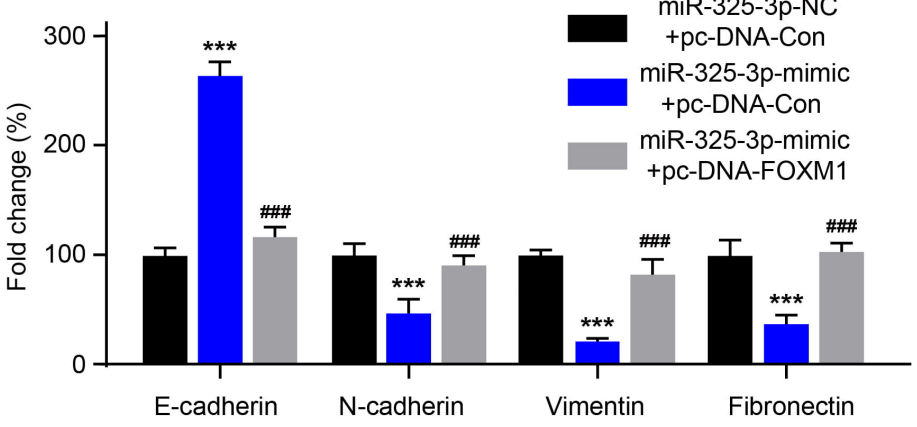

Fig. 6. MiR-325-3p regulates migration and invasion in U87 cells by targeting FOXM1 expression. (A) Western blot analysis showed that upregulation of miR-325-3p decreased the expression of FOXM1, and this effect was reversed by transfection with pcDNA3.0-FOXM1 in U87 cells. (B-E) Functional rescue experiments with Transwell assays showed that pcDNA3.0-FOXM1 transfection restored the migration and invasion abilities of U87 cells transfected with miR-325-3p-mimic. (F) The expressions of EMT-related genes were detected after FOXM1 was overexpressed in U87 cells via western blot. Data are

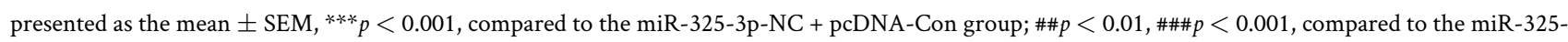
3p-mimic + pcDNA-FOXM1 group. 
A

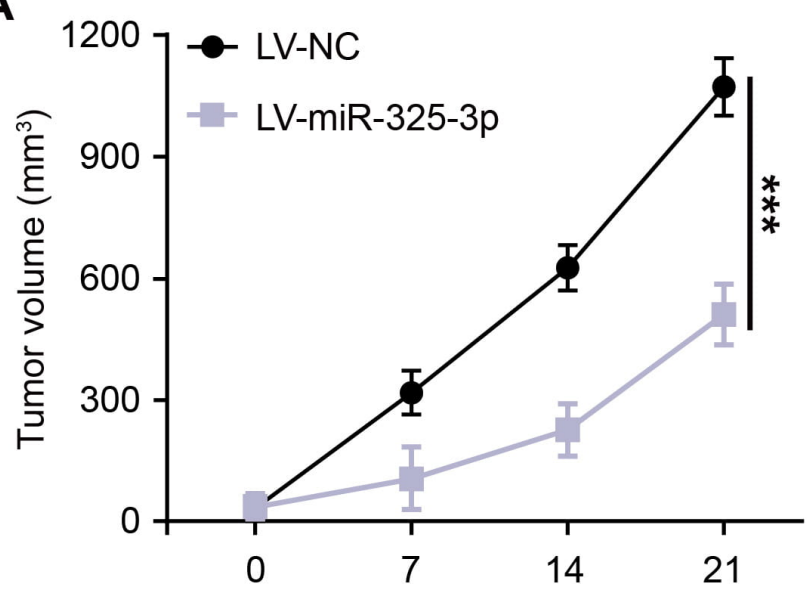

B

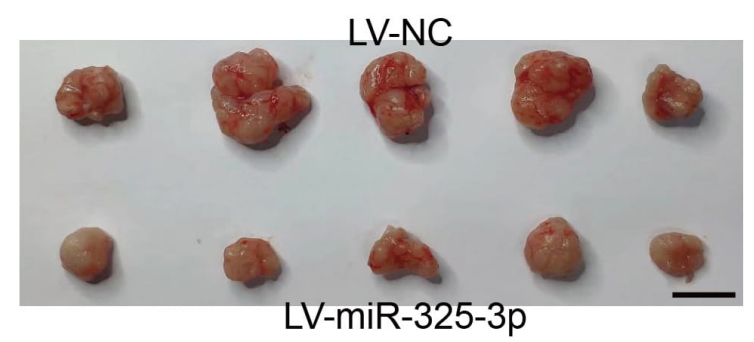

C

HE

LV-NC

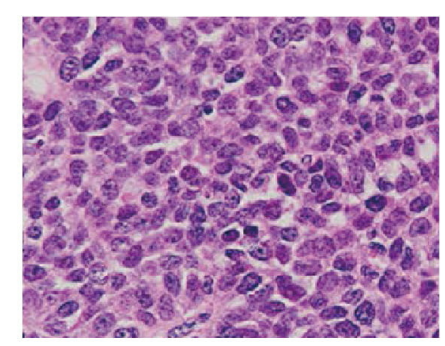

LV-miR-325-3p

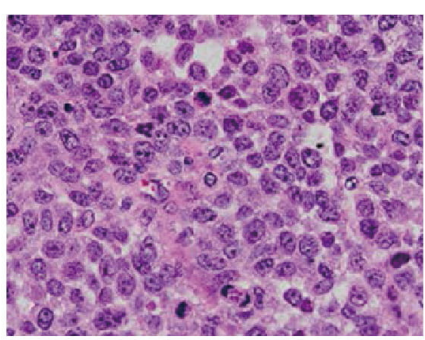

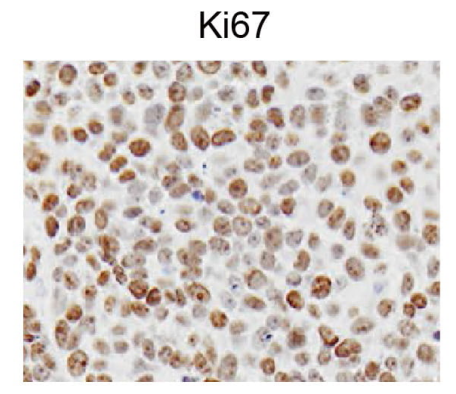

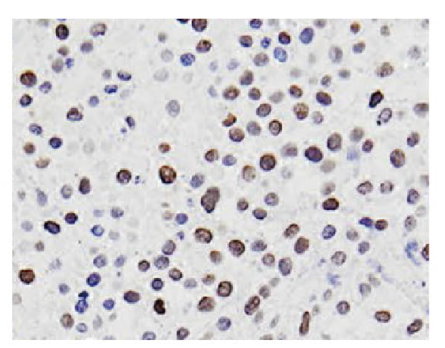

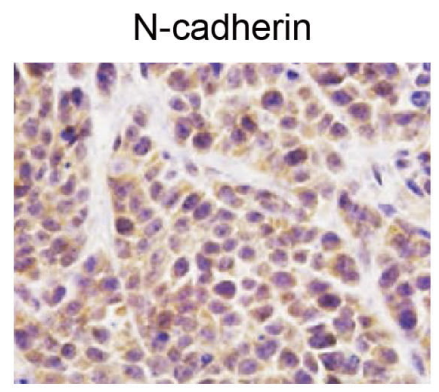

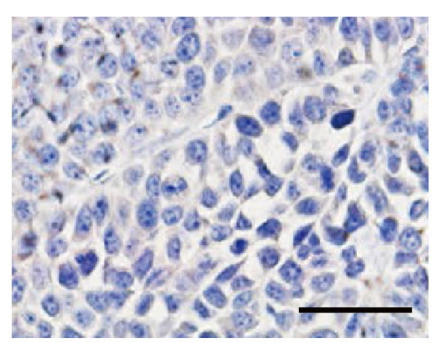

Fig. 7. MiR-325-3p repressed glioma growth in vivo. (A) Tumor volumes from the U87 cell xenograft model from the miR-325-3p-overexpressing group were significantly reduced. (B) Representative tumor images from the xenograft mice. (C) As indicated, representative H\&E staining and IHC images of Ki67 and $N$-cadherin in subcutaneous xenografts derived from cells. Scale bar: $100 \mu \mathrm{m}$. Data are presented as the mean \pm SEM, ${ }^{* * *} p<0.001$, compared to the LV-NC group.

and invasion in glioma U87 and SW 1783 cells using in vitro assays. These results provided elementary evidence for a role for miR-325-3p as a tumor-suppressor in glioma.

Similarly, it has been demonstrated that overexpression of miR-325-3p Inhibits proliferation and metastasis of bladder cancer cells [11] and miR-325 can also inhibit proliferation but induce apoptosis of $\mathrm{T}$ cells in acute lymphoblastic leukemia [20]. In hepatocellular carcinoma cells, miR-325$3 \mathrm{p}$ can inhibit cell proliferation and induce apoptosis in hepatitis B virus-related hepatocellular carcinoma [21]. The performance of miR-325-3p in regulating glioma cells migration and invasion implied an essential role for this miRNA in the mediation of glioma oncogenesis and tumor behavior, which is similar to previous studies mentioned above.
We further explored the potential mechanism by which miR-325-3p inhibited the invasion and migration in glioma and found FOXM1 as a potential target for miR-325-3p according to microRNA target databases. We found that FOXM1 was upregulated in glioma tissues and glioma cell lines compared to the control tissues and cells. Furthermore, statistical analysis showed a clear negative correlation between miR-325-3p and FOXM1. Our western blot data showed that miR-325-3p could reduce FOXM1 protein expression in vitro, and our dual-luciferase reporter assay confirmed an interaction between miR-325-3p and FOXM1 mRNA by directly targeting its 3'-UTR. Furthermore, in our rescue experiments, overexpression of FOXM1 countered the miR-325-3p-induced inhibitory effect on invasion and migration and metastasis-associated EMT pro- 
tein expression, further supporting an association between miR-325-3p and FOXM1. Functionally FOXM1 as a typical proliferation-associated transcription factor is required to execute the mitotic program and chromosome stability [16]. It is also significantly elevated in most human tumors and promotes tumorigenesis in many tissues, including pancreatic, esophageal, gastric, ovarian, and breast cancers $[17,18,22,23]$. Tissue microarray analysis revealed that FOXM1 expression was significantly higher in high-grade glioma than low-grade astrocytomas. The expression level of FOXM1 protein is directly related to the advanced grade, metastasis, and is negatively correlated with patient survival [24-26].

Several studies have also demonstrated that overexpression of FOXM1 promotes tumorigenicity, invasion, and angiogenesis of glioma cells [27-29]. However, in FOXM1 transgenic mice, no spontaneous brain tumors were observed [30], suggesting that FOXM1 overexpression alone does not induce gliomas or the presence of upstream regulatory mechanisms. Thus, it was important to determine the upstream miRNAs involved in the direct regulation of FOXM1, as these could represent potential biomarkers or therapeutical targets. We first confirmed the downregulation of miR-325$3 \mathrm{p}$ in glioma tissue and glioma cell lines. Therefore, upregulation of miR-325-3p could potently inhibit glioma cell proliferation and metastasis both in vitro and in vivo. Mechanistically, we found that the miR-325-3p/FOXM1 signaling pathway revealed a novel molecular mechanism for glioma progression suggesting that miR-325-3p could be a potential therapeutic target for malignant glioma. Finally, in our in vivo xenografted study, we confirmed again that miR-325-3p negatively inhibited tumor growth in mice.

\section{Conclusions}

MiR-325-3p was demonstrated to function as a tumor suppressor in glioma, at least in part, by targeting FOXM1. These findings may further elucidate the molecular mechanisms underlying glioma progression and provide a novel target and a better theoretical basis for new potential mechanisms involving the pathogenesis and molecular therapeutic strategy for glioma.

\section{Abbreviations}

miRNA, MicroRNA; CRC, colorectal cancer; GC, gastric cancer; EMT, epithelialmesenchymal transition.

\section{Author contributions}

HS conceived and designed the present study. HS and QJX performed the experiments and analyzed the data. HS and QJX interpreted the data and wrote the manuscript. All authors read and approved the final manuscript.

\section{Ethics approval and consent to participate}

Animals were raised and handled at the QJX at the Laboratory Animal Center of the Chongqing Medical Univer- sity. All animal experiments were carried out in accordance with the guidelines of the current institutional guidelines for the care and use of experimental animals and approved by the Animal Care Committee of Yongchuan Hospital of Chongqing Medical University. The glioma tissues were obtained with all participants' informed consent, and the research conducted was approved by the Ethics Committee of Yongchuan Hospital of Chongqing Medical University (2020108).

\section{Acknowledgment}

We thank two anonymous reviewers for their excellent criticism of the article.

\section{Funding}

This work was supported by funding from Yongchuan Hospital Affiliated to Chongqing Medical University (YJZQN201525).

\section{Conflict of interest}

The authors declare no conflict of interest.

\section{References}

[1] Barnholtz-Sloan JS, Ostrom QT, Cote D. Epidemiology of Brain Tumors. Neurologic Clinics. 2018; 36: 395-419.

[2] Anjum K, Shagufta BI, Abbas SQ, Patel S, Khan I, Shah SAA, et al. Current status and future therapeutic perspectives of glioblastoma multiforme (GBM) therapy: A review. Biomedicine \& Pharmacotherapy. 2017; 92: 681-689.

[3] Stewart LA. Chemotherapy in adult high-grade glioma: a systematic review and meta-analysis of individual patient data from 12 randomised trials. Lancet. 2002; 359: 1011-1018.

[4] Sanai N, Polley M, Berger MS. Insular glioma resection: assessment of patient morbidity, survival, and tumor progression. Journal of Neurosurgery. 2010; 112: 1-9.

[5] Alinezhad A, Jafari F. Novel management of glioma by molecular therapies, a review article. European Journal of Translational Myology. 2019; 29: 8209.

[6] Wang X, Liu C, Chen L, Zhang QC. RNA structure probing uncovers RNA structure-dependent biological functions. Nature Chemical Biology. 2021; 17: 755-766.

[7] Grimson A. Linking microRNAs to their targets. Nature Chemical Biology. 2015; 11: 100-101.

[8] Yue X, Schwartz JC, Chu Y, Younger ST, Gagnon KT, Elbashir $\mathrm{S}$, et al. Transcriptional regulation by small RNAs at sequences downstream from 3' gene termini. Nature Chemical Biology. 2010; 6: 621-629.

[9] Chengling L, Yulin Z, Xiaoyu X, Xingchen L, Sen Z, Ziming W, et al. MiR-325-3p, a novel regulator of osteoclastogenesis in osteolysis of colorectal cancer through targeting S100a4. Molecular Medicine. 2021; 27: 23.

[10] Sun T, Li K, Zhu K, Yan R, Dang C, Yuan D. SNHG6 Interacted with miR-325-3p to Regulate Cisplatin Resistance of Gastric Cancer by Targeting GITR. OncoTargets and Therapy. 2020; 13 : 12181-12193.

[11] Sun S, Liu F, Xian S, Cai D. miR-325-3p Overexpression Inhibits Proliferation and Metastasis of Bladder Cancer Cells by Regulating MT3. Medical Science Monitor. 2020; 26: e920331.

[12] Gan H, Lin L, Hu N, Yang Y, Gao Y, Pei Y, et al. KIF2C exerts an oncogenic role in nonsmall cell lung cancer and is negatively regulated by miR-32-3p. Cell Biochemistry and Function. 2019; 37: 424-431. 
[13] Zhang C, Li Y, Qin J, Yu C, Ma G, Chen H, et al. TMTBased Quantitative Proteomic Analysis Reveals the Effect of Bone Marrow-Derived Mesenchymal Stem Cell on Hair Follicle Regeneration. Frontiers in Pharmacology. 2021; 12: 658040.

[14] Zhu DP, Sun CX, Qian X. MST1 suppresses viability and promotes apoptosis of glioma cells via upregulating SIRT6 expression. Journal of Integrative Neuroscience. 2019; 18: 117-126.

[15] Xu LN, Wang SH, Su XL, Komal S, Fan HK, Xia L, et al. Targeting Glycogen Synthase Kinase 3 Beta Regulates CD47 Expression After Myocardial Infarction in Rats via the NF- $x B$ Signaling Pathway. Frontiers in Pharmacology. 2021; 12: 662726.

[16] Laoukili J, Kooistra MRH, Brás A, Kauw J, Kerkhoven RM, Morrison $\mathrm{A}$, et al. FoxM1 is required for execution of the mitotic programme and chromosome stability. Nature Cell Biology. 2005; 7 : 126-136.

[17] Kimura H, Sada R, Takada N, Harada A, Doki Y, Eguchi H, et al. The Dickkopf1 and FOXM1 positive feedback loop promotes tumor growth in pancreatic and esophageal cancers. Oncogene. 2021; 40: 4486-4502.

[18] Ai C, Zhang J, Lian S, Ma J, Győrffy B, Qian Z, et al. FOXM1 functions collaboratively with PLAU to promote gastric cancer progression. Journal of Cancer. 2020; 11: 788-794.

[19] Wijnenga MMJ, French PJ, Dubbink HJ, Dinjens WNM, Atmodimedjo PN, Kros JM, et al. Prognostic relevance of mutations and copy number alterations assessed with targeted next generation sequencing in IDH mutant grade II glioma. Journal of NeuroOncology. 2018; 139: 349-357.

[20] Wang F, Wang F, Zhang S, Xu X. MicroRNA-325 inhibits the proliferation and induces the apoptosis of $\mathrm{T}$ cell acute lymphoblastic leukemia cells in a BAG2-dependent manner. Experimental and Therapeutic Medicine. 2021; 21: 631.

[21] Zhang Z, Han Y, Sun G, Liu X, Jia X, Yu X. MicroRNA-325-3p inhibits cell proliferation and induces apoptosis in hepatitis $B$ virusrelated hepatocellular carcinoma by down-regulation of aquaporin 5. Cellular \& Molecular Biology Letters. 2019; 24: 13.
[22] Zhang Z, Tu K, Liu F, Liang M, Yu K, Wang Y, et al. FoxM1 promotes the migration of ovarian cancer cell through KRT5 and KRT7. Gene. 2020; 757: 144947.

[23] Zanin R, Pegoraro S, Ros G, Ciani Y, Piazza S, Bossi F, et al. HMGA1 promotes breast cancer angiogenesis supporting the stability, nuclear localization and transcriptional activity of FOXM1. Journal of Experimental \& Clinical Cancer Research. 2019; 38: 313.

[24] Zhang C, Han X, Xu X, Zhou Z, Chen X, Tang Y, et al. FoxM1 drives ADAM17/EGFR activation loop to promote mesenchymal transition in glioblastoma. Cell Death \& Disease. 2018; 9: 469.

[25] van den Boom J, Wolter M, Kuick R, Misek DE, Youkilis AS, Wechsler DS, et al. Characterization of Gene Expression Profiles Associated with Glioma Progression Using OligonucleotideBased Microarray Analysis and Real-Time Reverse TranscriptionPolymerase Chain Reaction. The American Journal of Pathology. 2003; 163: 1033-1043.

[26] Jiang L, Zhong M, Chen T, Zhu X, Yang H, Lv K. Gene regulation network analysis reveals core genes associated with survival in glioblastoma multiforme. Journal of Cellular and Molecular Medicine. 2020; 24: 10075-10087.

[27] Zhang X, LV Q, Huang Y, Zhang L, Zhou H. Akt/FoxM1 signaling pathway-mediated upregulation of MYBL2 promotes progression of human glioma. Journal of Experimental \& Clinical Cancer Research. 2017; 36: 105 .

[28] Zhang N, Wei P, Gong A, Chiu W, Lee H, Colman H, et al. FoxM1 Promotes $\beta$-Catenin Nuclear Localization and Controls Wnt Target-Gene Expression and Glioma Tumorigenesis. Cancer Cell. 2011; 20: 427-442.

[29] Cheng SX, Tu Y, Zhang S. FoxM1 promotes glioma cells progression by up-regulating Anxa1 expression. PLoS ONE. 2013; 8: e72376.

[30] Dai B, Pieper RO, Li D, Wei P, Liu M, Woo SY, et al. FoxM1B Regulates NEDD4-1 Expression, Leading to Cellular Transformation and Full Malignant Phenotype in Immortalized Human Astrocytes. Cancer Research. 2010; 70: 2951-2961. 